\title{
Rapid design of aircraft fuel quantity indication systems via multi-objective evolutionary algorithms
}

\author{
David Judt ${ }^{\mathrm{a}, 1}$, Craig Lawson ${ }^{\mathrm{a}}$, and Albert S.J. van Heerden ${ }^{\mathrm{a}}$ \\ ${ }^{a}$ Centre for Aeronautics, Cranfield University, College Road, Cranfield, MK43 OAL, UK.
}

\begin{abstract}
The design of electrical, mechanical and fluid systems on aircraft is becoming increasingly integrated with the aircraft structure definition process. An example is the aircraft fuel quantity indication (FQI) system, of which the design is strongly dependent on the tank geometry definition. Flexible FQI design methods are therefore desirable to swiftly assess system-level impact due to aircraft level changes. For this purpose, a genetic algorithm with a two-stage fitness assignment and FQI specific crossover procedure is proposed (FQI-GA). It can handle multiple measurement accuracy constraints, is coupled to a parametric definition of the wing tank geometry and is tested with two performance objectives. A range of crossover procedures of comparable node placement problems were tested for FQI-GA. Results show that the combinatorial nature of the probe architecture and accuracy constraints require a probe set selection mechanism before any crossover process. A case study, using approximated Airbus A320 requirements and tank geometry, is conducted and shows good agreement with the probe position results obtained with the FQI-GA. For the objectives of accessibility and probe mass, the Pareto front is linear, with little variation in mass. The case study confirms that the FQI-GA method can incorporate complex requirements and that designers can employ it to swiftly investigate FQI probe layouts and trade-offs.
\end{abstract}

Keywords: Evolutionary Algorithm; Multi-objective optimization; Aircraft Fuel System; Sensor System Design; Quantity Indication; Knowledge-based engineering

\section{Introduction}

The design and development process of complex airframe systems, such as the fuel system, requires close interaction between safety and performance analyses [1]. Within this process, performance analysis is a key activity, and the support of it has seen much development in terms of computational modelling and simulation capabilities. The development of these capabilities is driven by the need to consider as many design options as possible during early stage systems architecture definition and sizing. This is to ensure that no potentially high performing solutions are omitted [2-4].

One system that requires extensive design support is the fuel system. Its multiple subsystems fulfil the top-level functions of - storage, delivery, and quantity indication. The design of these is closely coupled to the aircraft geometry, with the wing geometry being the most influential, as it contains most of the system equipment and the integral fuel tanks [5]. Previous work has addressed this, through parametric formulations of wing and integral wing fuel tank geometries. These were then coupled with a multi-disciplinary design optimization framework for preliminary aircraft design, or used within knowledge based engineering (KBE) frameworks aimed at fuel system conceptual design [6]. KBE is a design philosophy in which geometry and performance modelling efforts are modular, parametric and make use of embedded design rules. This enables fast execution of design studies, from the aircraft to the airframe system level, with a higher level of detail than is traditionally the case [7].

The subsystem most dependent on the wing geometry is the fuel quantity indication (FQI) system. Typically, aircraft rely on capacitance-based fuel level gauging probes as part of a dual redundant and dual channel FQI architecture. In the traditional design process, only a small number of FQI probe placement architectures are investigated, with little consideration of the tank and FQI design relationships. Therefore, even though it is a mature subsystem, approaching its design with a KBE framework $[8,9]$ would provide new insights into FQI, fuel system and aircraft level trade-offs.

The academic literature on probe placement algorithms is sparse, as they are often part of the intellectual property portfolio of the airframe system supplier delivering the subsystem design and hence unpublished.

\footnotetext{
${ }^{1}$ Corresponding Author, E-Mail: david.judt@ cranfield.ac.uk
} 
This paper presents the investigations of one implementation approach to establish probe architectures that satisfy the accuracy and redundancy requirements. The method is aligned with KBE principles and uses a heuristic optimization routine for the FQI probe placement problem. A parametric automated link is maintained between the wing geometry and the FQI system design process. This provides substantial flexibility, as new or modified wing geometries can be analysed in a single workflow, without geometry data import and export issues.

The subsequent sections of this paper are organized as follows. Section 2 presents the FQI design problem and links it to the wider class of node placement problems and algorithms. Section 3 describes the FQI-GA algorithm and tests a range of crossover procedures. An application case study involving a re-design of the Airbus A320 FQI system is described in Section 4. The final section provides the conclusions from this research.

\section{FQI design problem description}

The FQI design process is heavily influenced by the types of sensors used within the system. The basic principles of the most widely used sensor technology, the capacitance probe, has changed little since its early presentation in the 1940s [10]. In an abstract sense, a single probe represents a means to establish the fuel level at a specific point in the tank. Level measurements from several sensors establish a fuel surface geometry which, is translated into a volume by using known tank geometry information. Eventually the fuel mass is established, with the help of a fuel density measurement.

The traditional design processes are well described and a set of requirements for the probe placement can be summarized from $[11,12]$ as:

- Define a minimum number of probes consistent with the method of gauging and the requirements to minimize weight and cost, and maximize reliability.

- Accuracy requirements (per attitude, fill state and flight state) for a complex tank are to be satisfied with a number of placed probes.

- Maximize the gaugeable fuel.

- Maintain probe - structure clearances, to minimize fringing and water bridging effects, and to safeguard against thermal movements.

- Establish probe locations that are compatible with structure mounting interfaces.

- Ensure that probe positioning does not contribute to fouling of fuel system components.

- Avoid potential water collection areas.

- Ensure repeatability in production.
Computing the tank fuel volume from probe measurements has been approached by different means. In early designs, the analogue summation of the capacitance readings from each probe is scaled with the tank's height- volume relationship. Probes can then also be profiled, i.e. have a non-constant diameter along the probe length, to accommodate the non-linear nature of the height-volume curve $[10,13]$. The curve also changes with aircraft attitude, acceleration or tank structure deflections, requiring a compromised profiled probe selection [14]. This makes the probe profiling approach inherently inaccurate for tanks with complex geometries.

In modern approaches, the computation is performed digitally. From a set of probe measurements, a software program establishes fuel volume via a nonlinear fitted model of the heightvolume relationship, or a stored tank geometry model [13]. Models and data specific to attitudes and flight conditions, can be stored and selected as required. The flight attitude is a key input to the computation process. It is either established through a data connection with the air data/inertial reference systems (ADIRS) or calculated from a plane formed by the submerged length measurements of three active probes. Fig.1 presents a typical dual redundant FQI architecture for a single tank and the operational states for capacitance probes. A placed capacitance probe is either inactive (fully submerged or dry) or active (partially submerged). A single active probe is typically enough to establish a tank volume if the current flight attitude is received from the ADIRS.

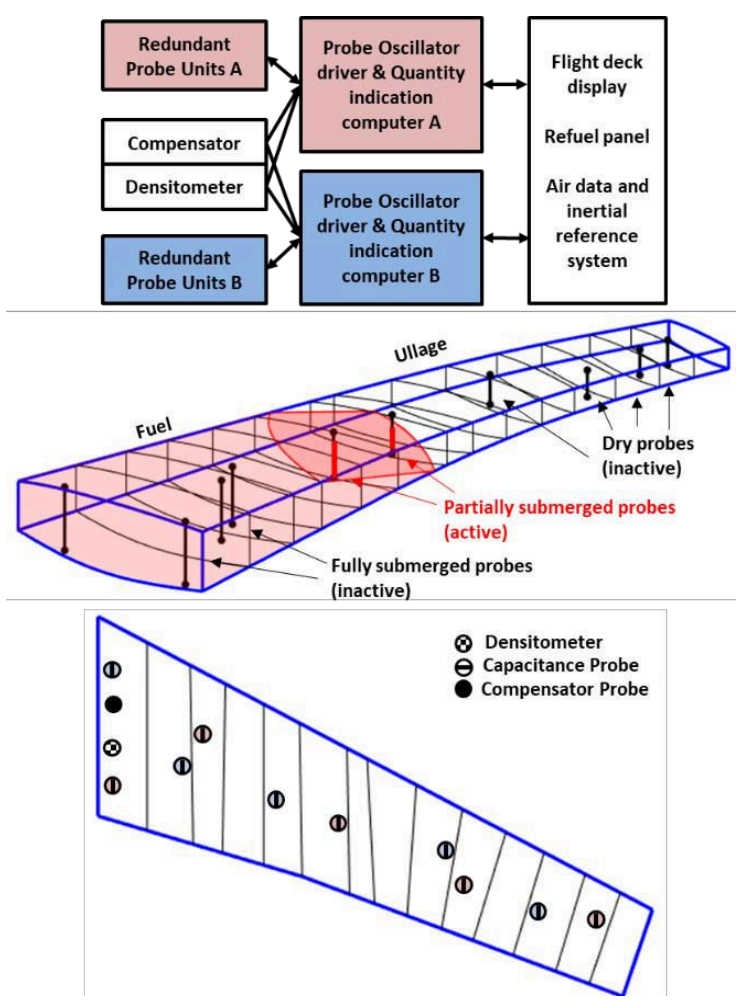

Fig.1. FQI Architecture and Probe operation. An active probe cuts across the fuel-ullage interface surface. 
The FQI probe placement design problem is part of a wider class of engineering problems referred to as node placement problems (NPP). Applications, such as wind farm layout design [15], wireless sensor placement [16] and structural health monitoring sensor networks [17], are typical examples of NPPs. Such problems require an unknown number of nodes to be placed to cover a defined area [18], achieve a degree of coverage redundancy [19], or maximize power extraction over a given area and wind-profile [20]. In Table 1, an overview of comparable NPP design problems and their design and optimization variables are provided.

In NPPs, the main property information per node is its $\mathrm{x}$ and $\mathrm{y}$ position in an area, bound by an upper and lower limit. For the FQI problem, additional properties, such as probe mass and an access metric are continuous variables, mapped directly to nodes. These are comparable to properties, such as transmitted power for a sensor node, or wind turbine hub height in NPPs [15]. However, other aspects of the FQI probe placement problem (FQI NPP) make it unique and arguably more challenging than past studied NPPs. These aspects include:

- There is a strong non-linear and discontinuous relationship between probe position and tank study surface coverage. This can lead to sudden changes in fitness for even small changes in probe position. Other NPPs have more gradual changes in fitness, such as the wake impact diminishing with spacing distance of turbines, or obstructions causing coverage variations when sensor positions change.

- Three related solutions to the NPP are required, in the form of the two independent probe sets and the single combined probe architecture. This adds a combinatorial dimension to the problem, not previously studied in NPPs.

Mixed design spaces of continuous and combinatorial nature, with discontinues objective functions, are typical in problems addressed by heuristic search methods, such as evolutionary algorithms (EA).

Table 1: Overview of FQI comparable NPP design problems.

\begin{tabular}{|l|l|l|l|l|}
\cline { 2 - 5 } \multicolumn{1}{c|}{} & $\begin{array}{l}\text { Wind farm design } \\
{[15,20,21,22]}\end{array}$ & $\begin{array}{l}\text { Wireless Sensor } \\
\text { Networks [16,19,18] }\end{array}$ & $\begin{array}{l}\text { Structural Health } \\
\text { Monitoring sensor } \\
\text { networks [23,24] }\end{array}$ & $\begin{array}{l}\text { FQI probe } \\
\text { placement }\end{array}$ \\
\hline Constraints & $\begin{array}{l}\text { Turbine proximity, } \\
\text { Wind conditions }\end{array}$ & $\begin{array}{l}\text { Sensor network } \\
\text { pattern }\end{array}$ & $\begin{array}{l}\text { Measurement } \\
\text { accuracy, } \\
\text { Probe proximity }\end{array}$ \\
\hline Objectives & $\begin{array}{l}\text { Installation cost, } \\
\text { Generated power }\end{array}$ & $\begin{array}{l}\text { Static coverage, } \\
\text { Energy consumption, } \\
\text { Coverage redundancy }\end{array}$ & $\begin{array}{l}\text { Probability of } \\
\text { damage detection, } \\
\text { Maximum area } \\
\text { coverage }\end{array}$ & $\begin{array}{l}\text { System mass, } \\
\text { Probe access }\end{array}$ \\
\hline $\begin{array}{l}\text { Design } \\
\text { variables }\end{array}$ & $\begin{array}{l}\text { Turbine position, } \\
\text { No. of turbines, } \\
\text { Turbine type and height }\end{array}$ & $\begin{array}{l}\text { Sensor position, } \\
\text { No. of sensors, } \\
\text { Sensing range }\end{array}$ & $\begin{array}{l}\text { Sensor position, } \\
\text { No. of sensors, } \\
\text { Sensor parameters }\end{array}$ & $\begin{array}{l}\text { Probe position, } \\
\text { No. of probes, } \\
\text { Probe set members }\end{array}$ \\
\hline Interactions & Turbine wake & Obstruction regions & $\begin{array}{l}\text { Structural boundary } \\
\text { signal reflections }\end{array}$ & $\begin{array}{l}\text { Tank study surface } \\
\text { case coverage }\end{array}$ \\
\hline
\end{tabular}

EAs use populations of solutions, in combination with variation and selection operators, to increase the mean fitness of the population over several generations [25,26]. Various engineering design problems have been approached with EAs, from the domains of energy and transport networks [27,28], to task scheduling [29,30] and aerospace [31-35]. Most real-world engineering optimization applications are of a constraint, multi-objective nature, and EAs should establish a set of Paretodominant solutions, which approximate the true front accurately in a region of interest to a decision maker.

Early algorithms, such as NSGA-II [36] and SPEA [37], made use of Pareto-ranking, crowding distance metrics and dominance strength ranks, to achieve evenly spaced and advancing Pareto fronts. An alternative has been proposed with MOEA/D [38], which decomposes the optimization problem into a set of scalar vector functions in the objective space and concurrently optimizes each. Therefore, some control over the spacing and diversity of solutions on the final Pareto front is available. Similarly, NSGA-III predefines a set of reference points on a hyperplane in the objective space and couples them to a niching strategy [39]. It is shown to perform well for optimization problems with more than 5 objectives. Another popular approach is the generalized differential evolution 3 algorithm GDE3 [40]. This operates with a fitness domain adaptive mutation and crossover operator, which makes GDE3 less reliant on control parameter settings.

In the domain of NPPs, genetic algorithms $[41,42]$ have been applied frequently. Since the solution representations to NPPs can be of variablelength, also referred to as metameric [43], special consideration when designing cross-over, mutation and selection mechanisms are needed to accommodate offspring generation, and prevent premature length convergence and solution bloat [44]. A flexible GA algorithm proposed by Zhang et al. [15], does not discretize the node position space, which has been typical in previous implementations. 
Instead, a subarea-swap crossover procedure ensures variable number of nodes are inherited by individuals.

Based on the success of heuristic optimization methods, for NPPs and due to the unique requirements of the FQI NPP, a new crossover procedure and constraint handling approach for a genetic algorithm is required.

\section{Genetic algorithm implementation for the FQI NPP}

\subsection{FQI NPP formulation}

Establishing fuel-ullage interface surfaces for complex 3D tank geometry data is computationally intensive and undesirable to be in the loop for an optimization process. This can be avoided by converting the continuous geometry space of the tank into a finite, discrete set. This enables computing grid cell data prior to executing the optimization routine, therefore decoupling it from the tank study execution.

With this approach, the tank grid data set constitutes the main set of information on tank study fuel surfaces, probe height, distances from access panels, and accuracy of the grid cell over the fuel surface cases. From this, an optimizer has to recompute constraint and objective values of cell combinations only.

The grid data set is summarised in Table 2 and the projection process for the tank study surface data is visualized in Fig.2.

Table 2: Grid cell information.

\begin{tabular}{|l|l|l|l|}
\hline ID & Grid Cell Data & Variable & Size \\
\hline 1 & Tank study surfaces & $\mathcal{T}$ & $N_{\text {cells }} * N_{\text {cases }}$ \\
\hline 2 & Probe height at cell & $\mathcal{H}$ & $N_{\text {cells }}$ \\
\hline 3 & $\begin{array}{l}\text { Min. distance from } \\
\text { access panel }\end{array}$ & $\mathcal{D}$ & $N_{\text {cells }}$ \\
\hline 4 & Grid case accuracy & $\mathcal{E}_{\text {grid }}$ & $N_{\text {cells }} * N_{\text {cases }}$ \\
\hline
\end{tabular}

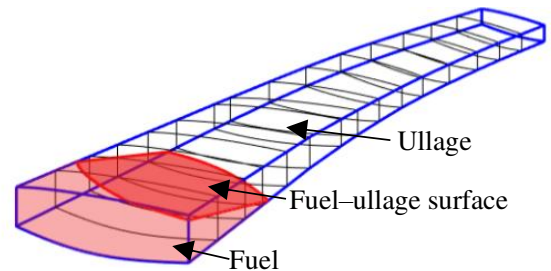

3D Fuel - ullage surface intersection for tank study case

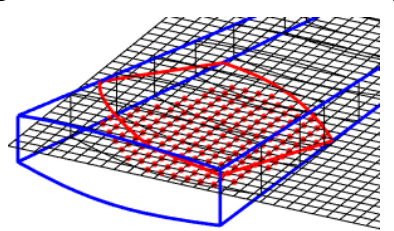

Fig.2. 3D surface projection along the $\mathrm{z}$-axis vector, onto an $\mathrm{x}-\mathrm{y}$ plane of 2D tank grid cells. Boolean grid data representing tank study surface $($ red $=1$, black $=0)$
The 3D tank geometry is projected on an $x-y$ plane along the $\mathrm{z}$-direction vector to define a tank grid, as shown in Fig.2.

This grid is the set $\mathcal{C}$ of cells with (x, y) position pairs:

$$
\mathcal{C}=\left\{(x, y)_{1},(x, y)_{2},(x, y)_{3}, \ldots,(x, y)_{N_{\text {cells }}}\right\}
$$

A grid cell can represent a probe position $p$ and a combination of probes form the probe set $\mathcal{P}$.

$$
\mathcal{P}=\left\{p_{1}, p_{2}, p_{3}, \ldots, p_{N_{\text {probes }}}\right\}
$$

The redundant probe sets $\mathrm{A}$ and $\mathrm{B}$ form an FQI architecture, $\mathcal{A}_{F Q I}$ :

$$
\mathcal{A}_{F Q I}=\left\{\mathcal{P}_{A}, \mathcal{P}_{B}\right\}
$$

A tank study produces a set of 3D fuel-ullage interface surfaces, $\mathcal{T}$ :

$$
\mathcal{T}=\left\{\mathcal{S}_{1}, \mathcal{S}_{2}, \mathcal{S}_{3}, \ldots, \mathcal{S}_{N_{\text {cases }}}\right\}
$$

Each surface $\mathcal{S}$ is projected on the same x-y plane and then defined by a set of Boolean entries per tank grid cell. The statement $b=1$ indicates that the cell is part of the fuel surface $\delta$ :

$$
\mathcal{S}=\left\{b_{1}, b_{2}, b_{3}, \ldots, b_{N_{\text {cells }}}\right\}
$$

where

$$
b \in\{0,1\}
$$

The accuracy requirements are represented by a set of volumetric errors $\mathcal{E}_{\text {req }}$ for each tank study case, where each volumetric error entry $r$, is calculated from the defined percentage errors for tank fill states and attitude:

$$
\varepsilon_{\text {req }}=\left\{r_{1}, r_{2}, r_{3}, \ldots, r_{N_{\text {cases }}}\right\}
$$

The accuracy set $\mathcal{E}_{\text {grid }}$ is computed for all tank study cases and grid cells.

$$
\begin{gathered}
\mathcal{E}_{\text {cell }}=\left\{e_{1}, e_{2}, e_{3}, \ldots, e_{N_{\text {cases }}}\right\} \\
\varepsilon_{\text {grid }}=\left\{\varepsilon_{\text {cell }, 1}, \mathcal{E}_{\text {cell }, 2}, \mathcal{E}_{\text {cell }, 3}, \ldots, \mathcal{E}_{\text {cell }, N_{\text {cells }}}\right\}
\end{gathered}
$$

The error value $e$ in $\mathcal{E}_{\text {cell }}$ is the accuracy of a probe in a grid cell and depends on the current submerged length and the probe upper and lower accuracy bounds. It is also a combination of probe, tank modelling and density measurement errors, as described in Section 5.

The tank height $h$ in each cell and the minimum distance $d$ of the cell from the closest access panel point are contained in sets $\mathcal{H}$ and $\mathcal{D}$, respectively.

$$
\begin{aligned}
\mathcal{H} & =\left\{h_{1}, h_{2}, h_{3}, \ldots h_{N_{\text {cells }}}\right\} \\
\mathcal{D} & =\left\{d_{1}, d_{2}, d_{3} \ldots d_{N_{\text {cells }}}\right\}
\end{aligned}
$$

The performance objectives for each $\mathcal{A}_{F Q I}$ are defined by the architecture mass $m_{F Q I}$ and the distance of probes from access panels, $d_{F Q I}$. For 
mass, the total architecture probe length is used as a proxy. This is effective, as the probe length-to-mass relationship is well established and often quoted by equipment suppliers [45]. However, representing accessibility with a simple access panel distance metric is only appropriate for preliminary design, but is insufficient during detailed design where component packaging, visibility and other ergonomic factors need to be evaluated.

$$
\begin{aligned}
m_{F Q I} & \rightarrow \sum_{j=1}^{N_{\text {probes }}} h_{\text {probe }, j} \\
d_{F Q I} & =\sum_{j=1}^{N_{\text {probes }}} d_{\text {probe }, j}
\end{aligned}
$$

where

$$
\begin{aligned}
& h_{\text {probe }, j} \in \mathcal{H} \\
& d_{\text {probe }, j} \in \mathcal{D}
\end{aligned}
$$

The FQI probe placement optimization problem (FQI-NPP) is to minimize a range of objective functions for a probe architecture, $\mathcal{A}_{F Q I}=\left\{\mathcal{P}_{A}, \mathcal{P}_{B}\right\}$. Here, two objectives for mass and access panel distance are chosen, namely

$$
\begin{aligned}
& \min m_{F Q I}=f_{1}\left(\mathcal{A}_{F Q I}\right) \\
& \min d_{F Q I}=f_{2}\left(\mathcal{A}_{F Q I}\right)
\end{aligned}
$$

Subject to the constraint that the gauging error of $\mathcal{A}_{F Q I}, \mathcal{P}_{A}$ and $\mathcal{P}_{B}$, as established by $\mathcal{E}_{\text {grid }}$, is within the required error bounds for all $\varepsilon_{\text {req }}$, as follows:

$$
\begin{aligned}
g_{c 1}\left(\mathcal{E}_{\text {cell }}\left(\mathcal{A}_{F Q I}\right), \mathcal{E}_{\text {req }}\left(\mathcal{A}_{F Q I}\right)\right) & \leq 0 \\
g_{c 2}\left(\mathcal{E}_{\text {cell }}\left(\mathcal{P}_{A}\right), \mathcal{E}_{\text {req }}\left(\mathcal{P}_{A}\right)\right) & \leq 0 \\
g_{c 2}\left(\mathcal{E}_{\text {cell }}\left(\mathcal{P}_{B}\right), \varepsilon_{\text {req }}\left(\mathcal{P}_{B}\right)\right) & \leq 0
\end{aligned}
$$

A further constraint is that the minimum distance between probes in $\mathcal{A}_{F Q I}$ must be above a cut-off value $d_{\text {cutoff }}$, to prevent probes being too close to each other:

$$
\begin{gathered}
g_{c 3}\left(\mathcal{A}_{F Q I}\right)>d_{\text {cutoff }} \\
p_{i} \in C
\end{gathered}
$$

\subsection{Overview of the FQI-GA}

The FQI-GA algorithm is an elitist GA with a two staged fitness assignment approach, random parent selection and a FQI-NPP specific formulation of crossover and mutation. A block diagram overview of its processes is presented in Fig. 4.

\subsection{Genetic representation}

The representation of a solution to the FQI NPP problem takes the form of a set of grid cell identifiers. If the absolute $x-y$ position values are required, the cell identifier is related back to the set $\mathcal{C}$ of grid position pairs.

A population of solutions is a matrix of grid cell identifiers with rows of variable length, where each row is an FQI probe architecture. The probe sets $\mathrm{A}$ and $\mathrm{B}$ are defined by a vector, storing the set split points per solution, as shown in figure 3 .

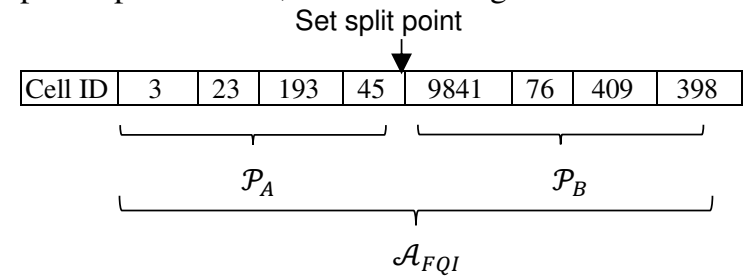

Fig. 3: Solution representation in the FQI-GA

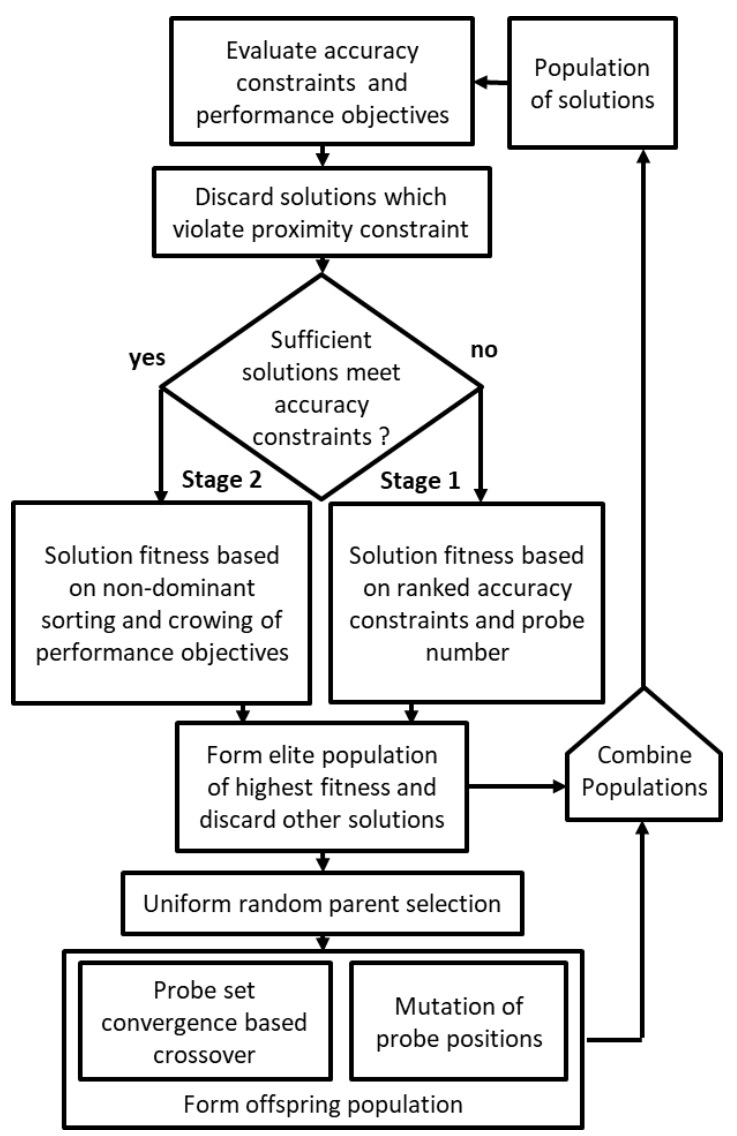

Fig. 4. Block diagram of the FQI GA process

\subsection{Constraint and Objectives handling}

The three accuracy constraints, as expressed in eq. (18-20), are translated into a single composite objective, through equal weighting. This is appropriate for the FQI problem, since the constraints are continuous [46]. For example, a probe architecture may only cover a certain number 
of fuel surface cases, leading to unmeasurable fuel states, which degrade the measurement accuracy. Therefore, as more surface cases are covered, the accuracy improves. The probe proximity constraint is considered through a death penalty method [46], i.e. solution sets with probes violating the constraint are discarded.

Meeting the accuracy constraints, while minimizing the probe mass and access, is a trade-off. It could be approached through a Pareto-based sorting method of the objectives with included penalty factor scaled constraint objectives. However, determining the penalty scaling factors is application-specific, and a different approach is therefore chosen.

Seok et al. [18], proposed a bi-population method, in which one set of constraint-satisfying and a second of partially-satisfying solutions are evolved in parallel. Rather than operating in parallel, the approach taken for the FQI problem operates in series to ensure only fully constraint-converged solutions can explore the performance objectives.

In the first stage, solutions with partially fulfilled constraints dominate the population. Their fitness is assigned through an equally weighted ranking of their three accuracy constraints and their number of probes in the solutions set. Including the probe number in the fitness assignment is a requirement, as its omission leads to premature convergence on solutions with high number of probes.

In the second stage, the elite population of accuracy-converged solutions is explored against the defined performance objectives. Solution fitness is now assigned with a standard non-dominant sorting mechanism, including crowding distance considerations [36]. Even though an NSGA-II type approach is outperformed by methods such MOEA/D and NSGA-III, it is simple in implementation and does not need a pre-definition of the reference domain for the Pareto-front.

\subsection{Selection and survival process}

As only the elite population produces offspring, its definition process, as described in section 3.4, already strongly drives selection. The parent selection method from this elite population is a uniform random selection. From the offspring and the elite population, a new population is formed, and all other solutions are therefore discarded.

\subsection{Crossover and mutation}

For the FQI problem, the transferable characteristics are the $(\mathrm{x}, \mathrm{y})$ position of the probes, the number of probes and their arrangement in sets. The FQI-GA offspring generation process is described by the block diagram in Fig. 5. First, the offspring solution length is determined by a uniform random selection of a value from the set $\mathcal{W}$ of solution lengths, where $N p_{\mathcal{A}_{P 1}}$ and $N p_{\mathcal{A}_{P 2}}$ are the solution lengths of parent 1 and 2 respectively.

$$
\mathcal{W}=\left[N p_{\mathcal{A}_{P 1}}-1, N p_{\mathcal{A}_{P 1}}, \ldots, N p_{\mathcal{A}_{P 2}}, N p_{\mathcal{A}_{P 2}}+1\right]
$$

Therefore, the number of probes of the parent architectures, determines the number of probes of the offspring, but can also increase or decrease by one. This extension ensures that both larger and smaller probe solution sets can be generated from the initial randomly seeded population.

The convergence of the accuracy constraint values of eq. (19) and eq. (20), for the four parent sets $\mathcal{P}_{A 1}, \mathcal{P}_{B 1}, \mathcal{P}_{A 2}, \mathcal{P}_{B 2}$, are used to select candidates for crossover. If none have converged, all sets have uniform probability of being inherited. This solution set based selective crossover approach ensures that converged sets are more likely to be regenerated with the same probe configuration. This part of the process is marked as (A) in Fig. 5.

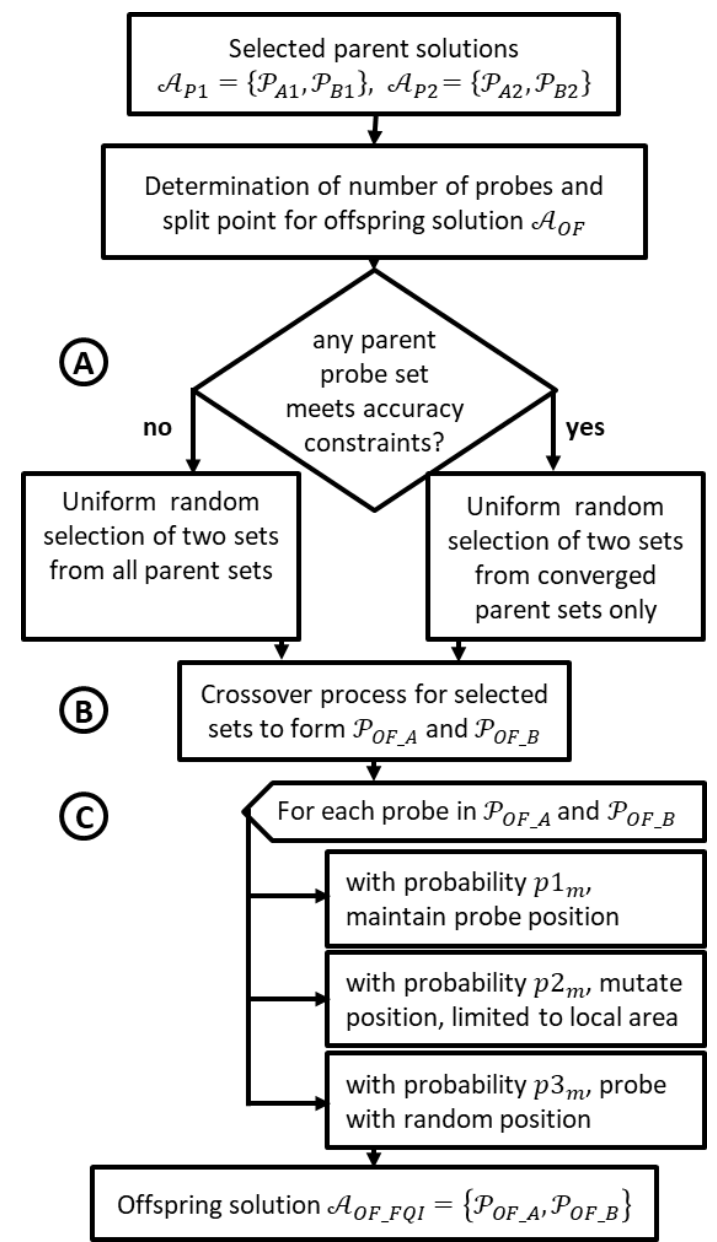

Fig. 5. Block diagram of the recombination method, using a constraint converged sorted set process (CC-SS)

A range of options have been investigated for the crossover process, marked as (B) in Fig. 5. In NPPs, the crossover has been addressed with a subareaswap operator in the past [15]. This operator randomly chooses two nodes and establishes the 
area swept by the radius of halve their Euclidian distance. Any nodes which fall in this area are swapped between the two parents to form an offspring node set. Other recombination mechanisms, such as single point crossover [25] and a performance-weighted ranked crossover were also implemented and investigated, with results presented in section 3.7.

Mutation, as described by part (C) in Fig. 5, either induces small positional variations for a probe position, or sets a new random position. The probability values, $p 2_{m}$ and $p 3_{m}$, control mutation with the options of a mutated position operation in the surrounding area of the parent probe, and a fully random new probe position, respectively. The third possibility is a maintained probe position, controlled by $p 1_{m}$. The probability of inheriting a fully random probe and a probe with a maintained or slightly modified position are EA parameters which tradeoff search diversity with convergence rate.

\subsection{Numerical experimental tests}

This section presents performance results for a set of experimental runs of variations of the FQI NPP GA. All experimental results are derived from the study of a single tank geometry, as visualized in Fig. 6. Algorithm parameter settings are constant for each variation investigated and are shown in Table 3. The algorithm variations studied focus on the crossover and parent selection processes only, while maintaining the NSGA-II like fitness assignment strategy for the Pareto set of solutions.

For each study case, the performance is measured using two hypervolume indicators (HV) [47], one for the performance objectives and the second for accuracy constraints. HV is a widely used metric, which captures the solution population's cardinality, accuracy and diversity in a single value, making it effective for comparisons [48]. The HV reference vector for both the median and $95 \%$ percentile confidence intervals of the $\mathrm{HV}$ results are calculated from a sample of six independent experiment executions.

In the first experiment, the recombination process feature of identifying and maintaining constraint converged sets separate from un-converged is tested. The baseline algorithm (FQI-GA-CC-SS) is as shown in Fig. 5, but with no operation taking place for step (B). The first modified process removes the decision step (A) and therefore produces offspring sets from any parent sent (FQI-GA-SS) with the mutation operation $(\mathrm{C})$ only.

The subarea-swap crossover operator is also tested, again in combination with constraintconverged set sorting (FQI-GA-CC-SAS) or operating on any two randomly selected sets (FQIGA-SAS). Even though care was taken to follow the implementation as described by Zhang et al. [15], it is important to note that the choice of software platform and difference in implementation can lead to significant performance differences [49].

Finally, two further crossover candidates, which operate after sorting sets by convergence, are tested. A single point crossover approach (FQI-GA-CC$\mathrm{SPC}$ ), with random crossover point selection and a probe performance metric crossover (FQI-GA-CCPPW). The probe metrics relate to the fuel surface cases a probe is uniquely active for, i.e. no other probe in the set is active. Metric $M_{1}$ expresses the number of unique surface cases covered as a ratio of total tank study cases $N_{\text {cases }}$. The second metric $M_{2}$ identifies the surface cases which have the highest ratio of fuel surface area $A_{k}$, unique $j$ to tank area $A_{\text {tank }}$.

$$
\begin{gathered}
M_{1, j}=\frac{N_{\text {cases, unique } j}}{N_{\text {cases }}} \\
M_{2, j}=\max \left(\frac{A_{\text {tank }}}{A_{k, \text { unique } j}}\right)
\end{gathered}
$$

Probes from the selected sets of the parents are ranked according to metrics $M_{1}$ and $M_{2}$. Their combined rank determines the order in which they will be inherited by an offspring set. The rationale for including such metrics into a crossover process is to encourage offspring probe sets, which maintain probes that are effective at covering the tank study fuel surface cases.

Table 3: FQI-GA parameters

\begin{tabular}{|l|l|l|}
\hline Symbol & Parameter & Value \\
\hline$N_{\text {gen }}$ & Number of generations & 1000 \\
\hline$N_{\text {pop }}$ & Population size & 500 \\
\hline$N_{\text {pareto }}$ & Elite population size & 50 \\
\hline$p 1_{m}$ & Probability to maintain position & $30 \%$ \\
\hline$p 2_{m}$ & Probability to mutate to local position & $60 \%$ \\
\hline$p 3_{m}$ & Probability to mutate to random position & $10 \%$ \\
\hline
\end{tabular}

Note: $p 1_{m}+p 2_{m}+p 3_{m}=100$
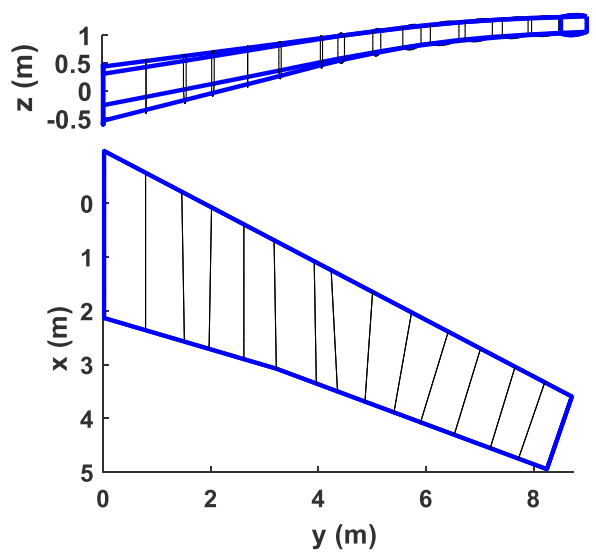

\begin{tabular}{|l|l|}
\hline Tank study cases, $N_{\text {cases }}$ & 990 (3 roll x 11 pitch x 30 fill states) \\
\hline
\end{tabular}

\begin{tabular}{|l|r|}
\hline Grid cell size & $0.05 \mathrm{~m} \times 0.05 \mathrm{~m}$ \\
\hline Tank grid cells, $N_{\text {cells }}$ & 7788 \\
\hline
\end{tabular}

Fig. 6. Tank geometry and parameters for the numerical experiments 


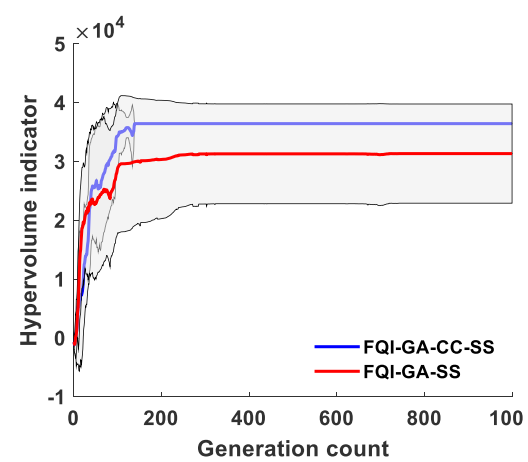

(a)

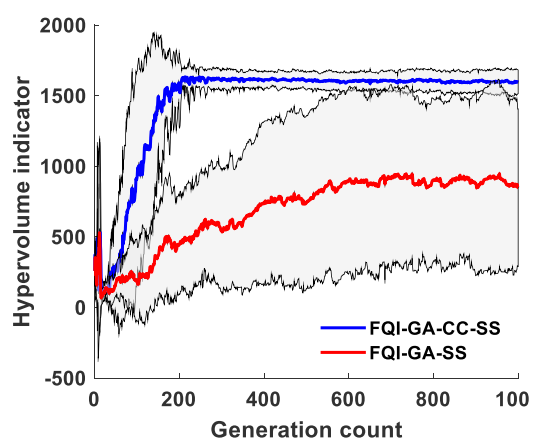

(d)

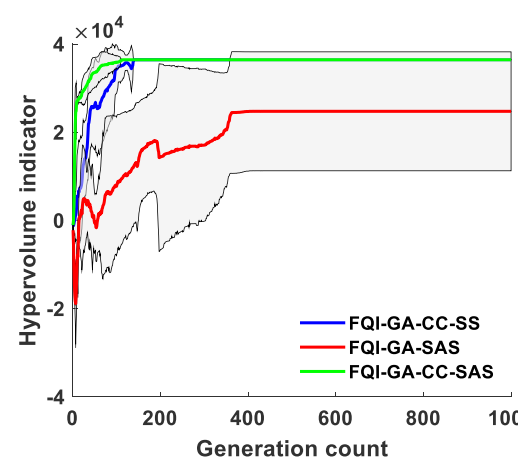

(b)

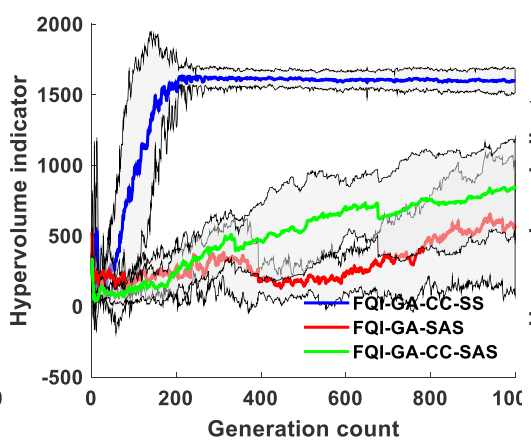

(e)

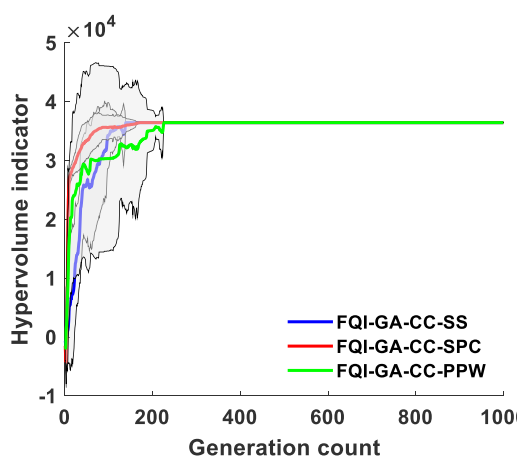

(c)

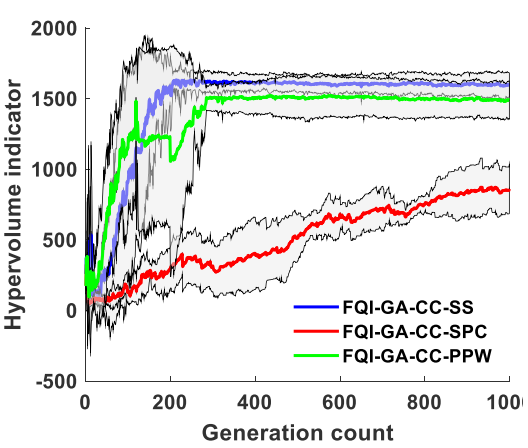

(f)

Fig. 7. Hypervolume indicator mean and $95 \%$ confidence interval (CI) results against generation count from the recombination variation experiments. (a-c) show the HV for the accuracy constraints. (d- f) show the HV for the performance objectives.

Table 4. HV results after $N_{\text {gen }}=1000$ for accuracy constraint and performance objectives

\begin{tabular}{|c|c|c|c|c|c|c|c|c|}
\hline & \multicolumn{4}{|c|}{ Hypervolume indicator of accuracy constraint fitness } & \multicolumn{4}{|c|}{ Hypervolume indicator of performance objectives } \\
\hline & Worst & Mean & Best & $95 \% \mathrm{CI}$ & Worst & Mean & Best & $95 \% \mathrm{CI}$ \\
\hline Random Search & 17674 & 19586 & 22563 & \pm 2094 & 31.21 & 73 & 130 & \pm 38 \\
\hline FQI-GA-CC-SS & 36450 & 36450 & 36450 & \pm 0 & 1537 & 1596 & 1718 & \pm 83 \\
\hline FQI-GA-SS & 18782 & 31357 & 36450 & \pm 8429 & 171 & 847 & 1479 & \pm 557 \\
\hline FQI-GA-SAS & 11183 & 24756 & 36450 & \pm 13485 & 185 & 555 & 1229 & \pm 476 \\
\hline FQI-GA-CC-SAS & 36450 & 36450 & 36450 & \pm 0 & 258 & 828 & 1268 & \pm 357 \\
\hline FQI-GA-CC-SPC & 36450 & 36450 & 36450 & \pm 0 & 607 & 860 & 1028 & \pm 173 \\
\hline FQI-GA-CC-PPW & 36450 & 36450 & 36450 & \pm 0 & 1281 & 1490 & 1611 & \pm 134 \\
\hline
\end{tabular}

Results in Fig. 7a, Fig. 7b and Fig. 7c show that making a distinction between non-converged and converged accuracy constraints sets is key to enable convergence, independent of the crossover process. This is due to the combinatorial nature of the solution, where specific sets of probes together fulfil a constraint.

The results of the different crossover processes show that both the subarea swap crossover and a single point crossover are performing poorly for the FQI NPP. The mixing of probes from two converged sets does not effectively lead to offspring sets which are both converged and explore the Pareto front well. An exception is the FQI-GA-CC-PPW crossover method, where probe metrics influence the selected probe to be inherited. However, as shown by the Fig. $7 \mathrm{f}$, and Table 4, even if such a crossover process is chosen, the baseline algorithm FQI-GA-CC-SS outperforms with only the use of mutation operations.
Even for the best performing crossover implementation, FQI-GA-CC-SS, the Pareto-front quality, as indicated by the HV mean and $95 \%$ CI could be improved. One option would be to use the $\mathrm{HV}$ for solution fitness assignment. Calculating the contribution each Pareto-optimal solution makes to the $\mathrm{HV}$ and combining it with a grid based objective space niching strategy, has been shown to be effective [50,51]. This does not require significant changes to the FQI-GA two-staged architecture, as would be the case with MOE/D or NSGA-III.

Finally, algorithm performance results presented here need to be investigated for grid cell size sensitivity. The chosen value of $0.05 \mathrm{~m}$ is based on typical physical probe size diameters and therefore represents the lower limit on grid cell size. 


\section{Application study}

\subsection{Analysis of the Airbus A320 FQI System}

Available data from the A320 FQI system and wing geometry [52-54] was compiled to re-create an approximation of the wing tank geometry, as shown in Fig. 8. The geometry is generated with a bespoke parametric representation for the wing, which could be replaced by other parametric aircraft geometry tools, such as OpenVSP [55]. The main purpose is to couple the FQI-GA method to a parametrically defined geometry, to enable its implementation in KBE frameworks.

Gauging accuracies are especially important in the ground attitude for accurate refuelling. ARINC 611-1 specifies typical requirements for large aircraft of $-0.5 \%$ under-read at empty and no overread, increasing linearly to $+/-1 \%$ at full tank [11]. The Airbus A340 gauging system has an accuracy of around $0.4 \%$ at empty and $1 \%$ at full [56]. Accuracies for the A320 are quoted as being between $+/-1 \%$ at empty and $+/-2 \%$ at full $[57,58]$. In order to have zero over-read at empty/unusable fuel conditions [59], FQI systems are bias corrected to shift their over and under read curves downwards.

Based on standard practice, three attitude scenarios were defined for the case study: ground, normal flight, and flight with extended roll and pitch attitude. Aircraft operations data sheets $[60,61]$ were used to define the roll and pitch attitude limits for the given scenarios. The extended scenario values were based on the A320 flight envelope protection limits for angle of attack and pitch, whereas normal flight values are based on typical climb and descent flight path angles. On the ground, typical runway slope limits were used to define the ground attitude range [61].

Given these data, the estimated A320 accuracy requirements for the attitude scenarios were defined as shown in Table 5. A standard accuracy degradation factor (DF) of three was used for cases where the failure of one redundant probe set had occurred [11].

The case study focuses on the inboard tank only, which runs from the root rib, until rib number 15 . Rib 2 has a set of flap valves, which only allow fuel to flow inboard. For the purpose of this case study, these flap valves have not been considered in the model.

The attitude envelope of $+/-10^{\circ}$ pitch and $+/-3^{\circ}$ roll over the tank fill state was discretized to conduct the tank study. Roll was selected to be represented by 3 cases, whereas pitch was discretized at 2-degree intervals, leading to 11 pitch cases. The tank fill state was varied at $3 \%$ steps, leading to 30 discrete cases, including empty and full. The empty and full cases correspond to the unusable fuel and max fill case of the tank, respectively. For the A320, these were approximated to be $0.1 \%$ and $98 \%$ of the tank volume. With these attitude and fill state step sizes, a total of 990 tank study cases are needed. Fig. 9 illustrates some of the resulting tank study fuelullage interface surfaces.

The total number of tank study cases, together with the chosen grid cell size, determines memory allocation requirements. For this case study, a combined parallel runtime of 30 minutes on an Intel i7-2600 CPU @ 3.4GHz using 8GB of RAM and Windows 7 , was sufficient.

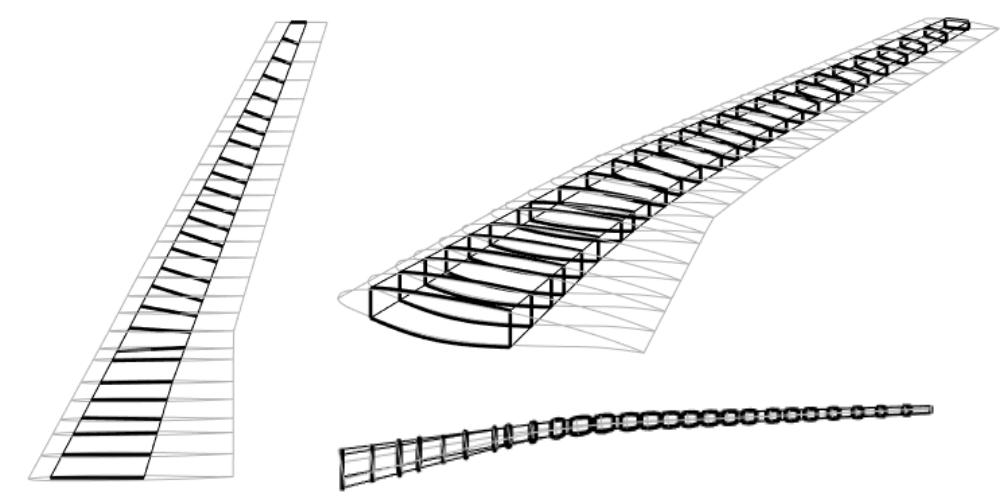

Fig. 8. A320 wing and tank geometry approximation.

Table 5: Approximated A320 accuracy requirements from [57,58].

\begin{tabular}{|c|c|c|c|c|c|c|}
\hline \multirow{2}{*}{ Mode } & \multirow{2}{*}{ DF } & \multicolumn{2}{|c|}{ Accuracy } & \multicolumn{3}{|c|}{ Attitude Range $\left({ }^{\circ}\right)$} \\
\hline & & $\mathrm{Hi}$ & Lo & Pitch & Roll & Case \\
\hline \multirow{3}{*}{ Normal } & \multirow{3}{*}{1} & & $-0.5 \% \mathrm{FF}-0.5 \% \mathrm{FOB}$ & $+/-3$ & \multirow{6}{*}{$+/-3$} & Ground \\
\hline & & $+1 \% \mathrm{FOB}$ & $-1 \% \mathrm{FF}-0.5 \% \mathrm{FOB}$ & $-3<<+5$ & & Normal \\
\hline & & $+1 \% \mathrm{FF}+1.5 \% \mathrm{FOB}$ & $-1 \% \mathrm{FF}-1.5 \%$ FOB & $+/-10<<+/-3$ & & Extended \\
\hline \multirow{3}{*}{ Degraded } & \multirow{3}{*}{3} & $30 \mathrm{FOP}$ & $-1.5 \% \mathrm{FF}-1.5 \% \mathrm{FOB}$ & $+/-3$ & & Ground \\
\hline & & $+3 \%$ FUB & $-3 \% \mathrm{FF}-1.5 \% \mathrm{FOB}$ & $-3<<+5$ & & Normal \\
\hline & & $+3 \% \mathrm{FF}+4.5 \% \mathrm{FOB}$ & $-3 \% \mathrm{FF}-4.5 \% \mathrm{FOB}$ & $+/-10<<+/-3$ & & Extended \\
\hline
\end{tabular}

$F O B=$ Fuel on Board, $F F=$ Full Fuel, $D F=$ Degradation factor 
The A320 wing FQI probe system consists of two sets of probes, each having their own in-tank wiring harness and a probe oscillator driver. Both sets provide data to the fuel quantity indication computers, which calculate the current fuel mass per tank from the probe readings, ADIRS inputs, and stored tank height-volume tables. Probes are generally non-vertical, which allows them to cover more fuel surface but increases their length and therefore weight. An approximated representation of the dual redundant FQI architecture [58,62], is shown in Fig. 10. Each set has six probes and can cover all measurement requirements for the extended flight envelope under degraded accuracy values. As the FQI-GA operates on a $2 \mathrm{D}$ projection grid, the angled probes from the A320 are approximated with a vertical probe. In the optimal re-design approach, an FQI architecture redundancy is integrated by requiring two probe sets to independently fulfil the accuracy requirements. This makes the approach specific to a dual redundant FQI design philosophy, which is common in modern aircraft, as seen in the A320 example, but not explicitly required by civil certification standards.

Table 6: Error sources included in error modelling.

\begin{tabular}{|l|l|l|c|}
\hline $\begin{array}{l}\text { Error } \\
\text { source }\end{array}$ & Value & Type & Symbol \\
\hline $\begin{array}{l}\text { Density } \\
\text { error }\end{array}$ & $\begin{array}{l}\text { 0.4\% of density } \\
\text { indication }\end{array}$ & Random & $e_{d e}$ \\
\hline $\begin{array}{l}\text { Tank } \\
\text { modelling }\end{array}$ & $\begin{array}{l}0.2 \% \text { of max tank } \\
\text { volume }\end{array}$ & Bias & $e_{V, t}$ \\
\hline $\begin{array}{l}\text { Probe } \\
\text { error }\end{array}$ & $\begin{array}{l} \pm 0.5 \% \text { (dry) linearly } \\
\text { increasing to } \\
\pm 1.6 \% \text { (full) of probe } \\
\text { indication }\end{array}$ & Random & $e_{L B}, e_{U B}$ \\
\hline $\begin{array}{l}\text { No } \\
\text { coverage }\end{array}$ & $\begin{array}{l}\text { Non-measurable } \\
\text { volume between two } \\
\text { probes }\end{array}$ & Bias & $e_{V, c}$ \\
\hline
\end{tabular}

The gauging accuracy of a probe set, or architecture is calculated by considering three error sources: density measurement, tank modelling, and probe indication errors. For each tank analysis case, the levels of submersion of probes in the architecture varies, leading to changes in total gauging accuracy. A fourth error term is required in the case of a nonmeasurable volume, due to no probes being active for a tank. This is visible in Fig. 11, in which some cases are not covered by the probes and a resulting high spike in gauging error results. A summary of the error sources and their estimated values based on the available literature $[63,45]$, is presented in Table 6.

The total gauging mass error $e_{T}$ is the arithmetic combination of errors, as shown in eq. (26-28). The volume error for a probe is based on its length $l_{p}$, wetted length $l_{p, s}$, measurement error $e_{p, z}$ and the gradient of volume change per height $\frac{d V}{d z}$ of the corresponding tank section. The volume error is combined with a density error $\varepsilon_{d e n}$ and expressed as a ratio of maximum fuel mass, shifted by the bias term $\Delta_{F Q I, b}$.

$$
\begin{gathered}
e_{p, z}=\left( \pm e_{L B}+l_{p} *\left(\Delta e_{U B-L B}\right)\right) * l_{p, s} \\
e_{V, p}=e_{p, z} * \frac{d V}{d z} \\
e_{T}=\frac{\left( \pm e_{V, t} \pm \mathrm{e}_{V, p} \pm e_{V, c}\right) *\left(\rho \pm \varepsilon_{d e n}\right)}{V_{T} * \rho}-\Delta_{F Q I, b}
\end{gathered}
$$

Some tank study cases have multiple active probes. Each has its own volume error magnitude, depending on its wetted length and local volume/height gradient. The probe with the lowest error value is chosen as active for this case. Finally, all gauging errors are shifted with a system-bias, equal to the over-read value at the zero useable fuel level. This is a certification requirement for large civil aircraft under $\$ 25.1337$ [59].

There is a relation between accuracy estimations and the tank geometry and fuel surface calculations. For example, tank geometries with low dihedral angles, may require more roll angle steps, as the fuel surface changes more drastically compared with a high dihedral wing. In addition, the chosen discretization step size for the fuel state has a direct impact on the probe accuracy calculation in the case of a non-covered fuel surface. This is because the error term $e_{V, c}$ depends on the next case at which a probe is active, which may be closer or further, depending on the fill state step size. Finally, as the tank volume and surface calculations are coupled, the simplified geometry of the tank structure and the omission of internal fuel system components can render volume estimates inaccurate, which impacts the probe accuracy estimations.

Notwithstanding these potential modelling uncertainties, Fig. 11 shows the A320 tank study accuracy results for the two independent sets of probes and the nominal case where all probes are active. For the three attitude envelope cases, the highest over and under-read results are plotted as a boundary around the rest of the non-critical cases. This boundary can then be compared to the requirements, represented by the dashed lines.

The results show that, for the assumed error source magnitudes, the specified degraded accuracy limits were achieved. For the nominal limits of ground and normal flight attitudes, the under-read error between the 25 and $40 \%$ tank fill states was marginally exceeded as, indicated in Fig. 11. 

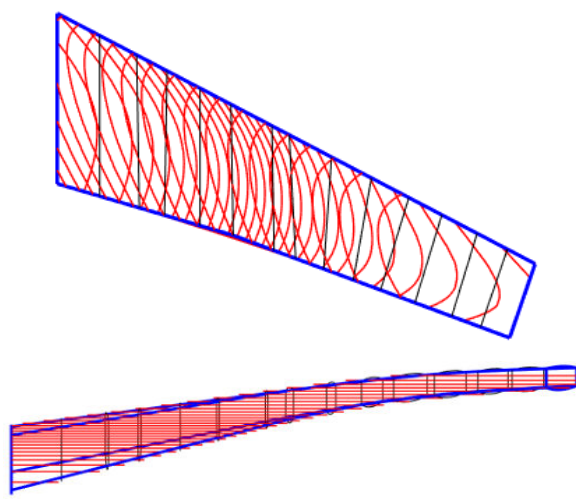

a) Fill state cases at $0^{\circ}$ pitch and $0^{\circ}$ roll angle
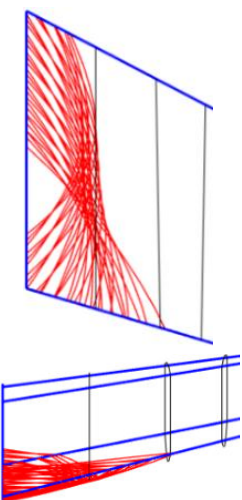

b) Pitch and roll angle cases at zero fill state

Fig. 9. Tank study results. The red lines represent contours of example fuel-ullage interface surfaces.
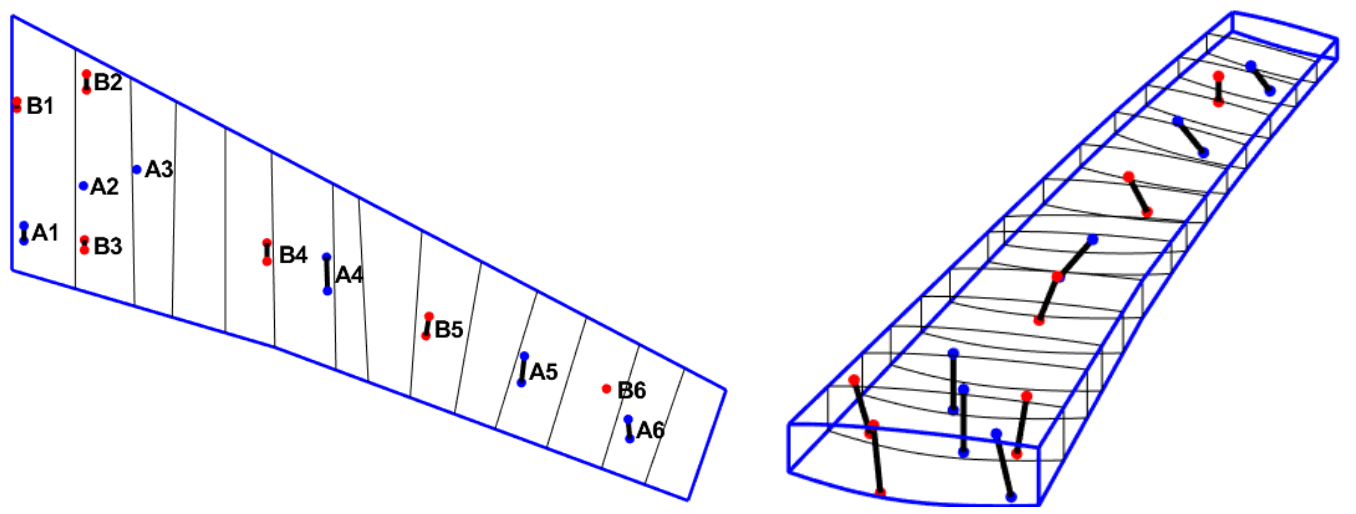

Fig. 10. Approximated A320 probe positions and directions.
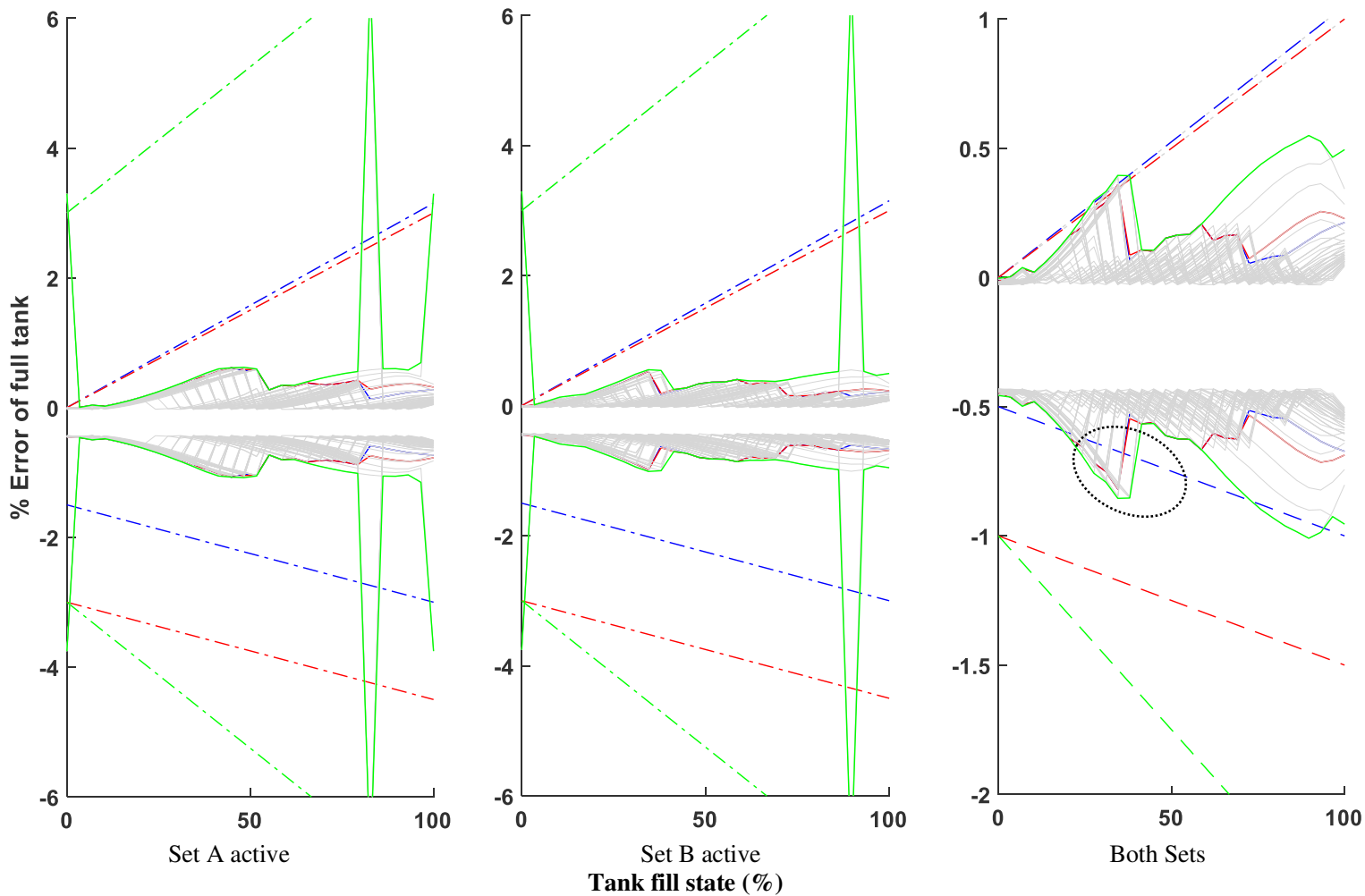

Requirement

Tank study cases

Ground evelope

Normal evelope

Extended evelope

Fig. 11. A320 tank study accuracy results for redundant probe sets and full architecture. 


\subsection{Optimal FQI probe placement redesign and comparison with the A320 baseline}

Given the case study geometry data and accuracy requirements, an overall satisfactory agreement between requirements and achieved accuracy was shown for the reconstructed A320 FQI architecture. Subsequently, this design is the baseline reference for comparisons with re-designs produced by the FQI-GA under equivalent requirements and constraints. Three studies were conducted, as shown in Table 7, with the same algorithm parameter settings from Table 3.

Table 7: A320 FQI probe placement redesign studies

\begin{tabular}{|l|l|}
\hline Study & Performance Objective(s) \\
\hline A & Minimize mass \\
\hline B & Maximize access \\
\hline C & Minimize mass \& maximize access \\
\hline
\end{tabular}

The resulting probe architectures from studies A and $\mathrm{B}$ are shown in Fig. 15. The optimization achieves a total probe count reduction of four for both. The probe count is driven by the accuracy constraints rather than the performance objectives and therefore is equal in both studies, whereas the probe positions varies. The reduced probe count leads to reduced overlap coverage of tank study fuelullage interface surface cases. This is illustrated in Fig. 12 and Fig. 13 for study A, with coverage for redundant set A shown only. Coverage is displayed for three single roll angle cases and a range of pitch angles and fill states. In the 0 to $50 \%$ fill state range, the coverage is provided by the $\mathrm{A} 320$ probes $\mathrm{A} 1, \mathrm{~A} 2$ and $\mathrm{A} 3$, located in the tank rib bays (RB) 1, 2 and 3 . Their combined coverage can be achieved by probes $\mathrm{AA} 1$ and AA2, which are located in RB1 and RB3.

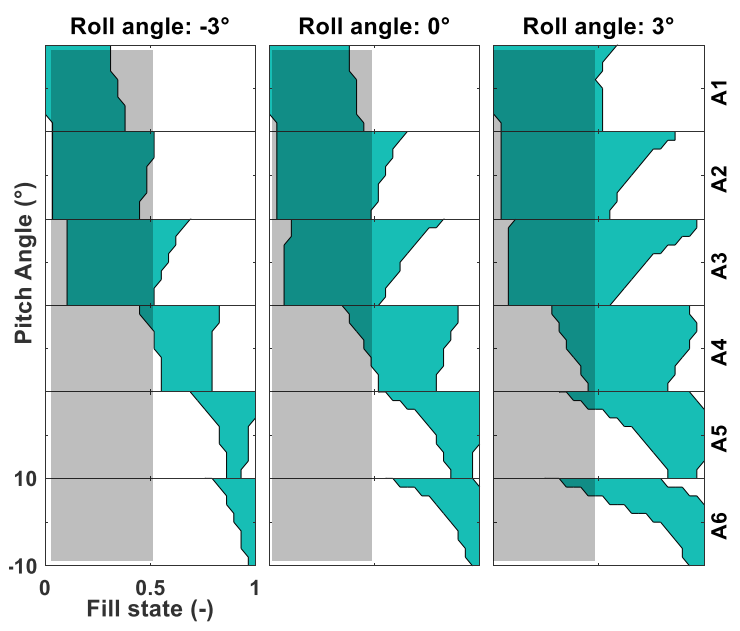

Fig. 12. Tank study coverage for the A320 and the study A resulting probes of set $\mathrm{A}$.

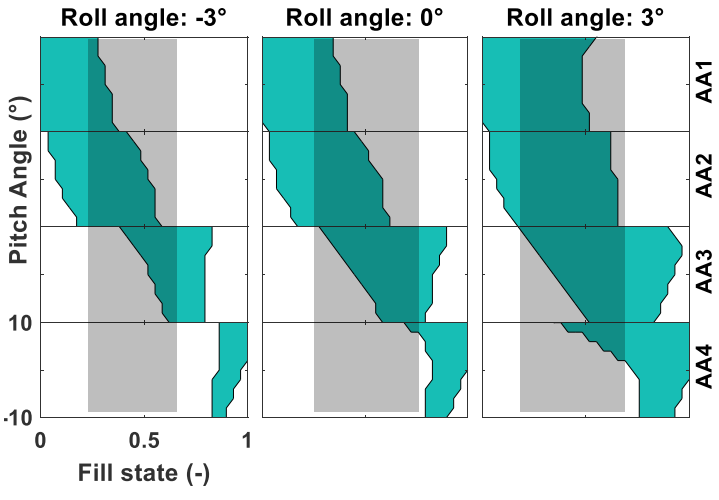

Fig. 13. Tank study coverage for the study A resulting probes of set A.

All individual probe coverages are combined to form the probe set coverage, as shown in Fig. 14. Overlap is reduced throughout the tank study cases, with the peak values reducing from 6 to 4 . There was some increase in areas of non-coverage, such as the $90-100 \%$ fill state at pitch angles above $-8^{\circ}$. However, this non-coverage does not lead to a violation of the accuracy requirements, as solutions from the FQI-GA are fully constraint-converged.

A simplification, which impacts the probe count for the re-design study, is the omission of the flap valves located in rib 2. Flap valves only allow fuel to flow unidirectional through a rib. Therefore, in the case of the A320, with a negative roll angle two fuel surfaces would form for the inboard tank; one located in RB1 and the second in the rest of the tank. This requires additional probes in RB2 in order to measure this surface at low fuel states, which are present in the A320 arrangement.

In the re-design studies, it is assumed that accuracy, rather than tank study case coverage requirements are to be met. However, it is possible that the A320 FQI probe placement requirements were based on coverage and accuracy, resulting in a design with more probes.

A probe position comparison is presented in Fig. 15 for studies A and B. In study A, probes were forced towards the front spar, where their length reduced due to the tank shape. Probe length can vary by as much as $0.5 \mathrm{~m}$ between the front and rear spars, at RB2. In contrast, for study B, probes are located towards the access panels, to minimize the access objective. Because the access panels are located around the thickest part of the rib bays, the resulting architectures have longer probes. 


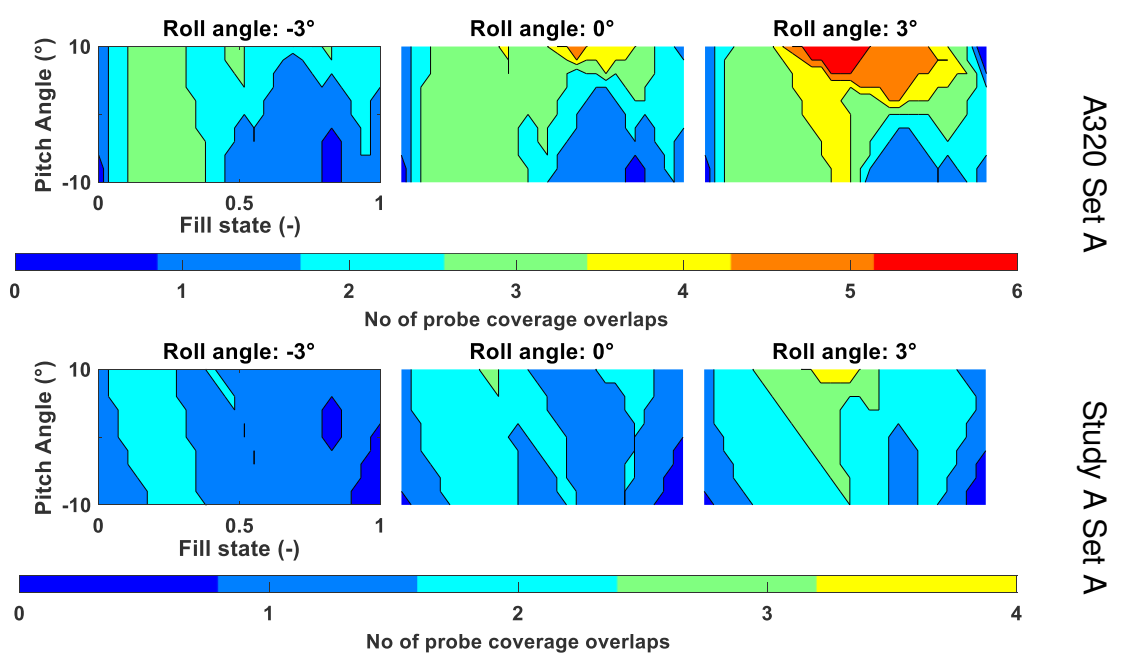

Fig. 14. Tank study coverage comparison for one probe set of the A320 and the FQI-GA study A.

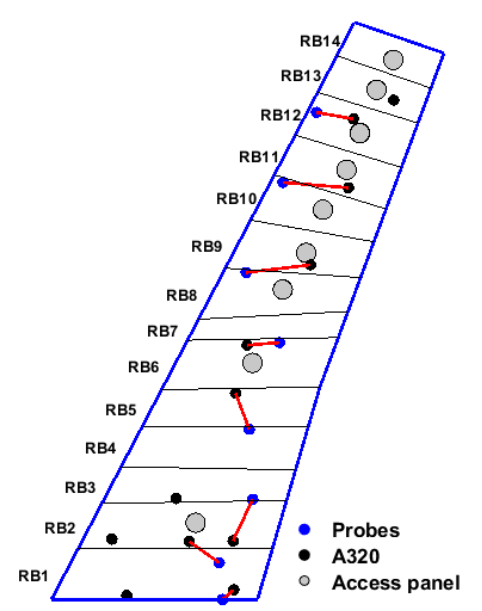

Study A

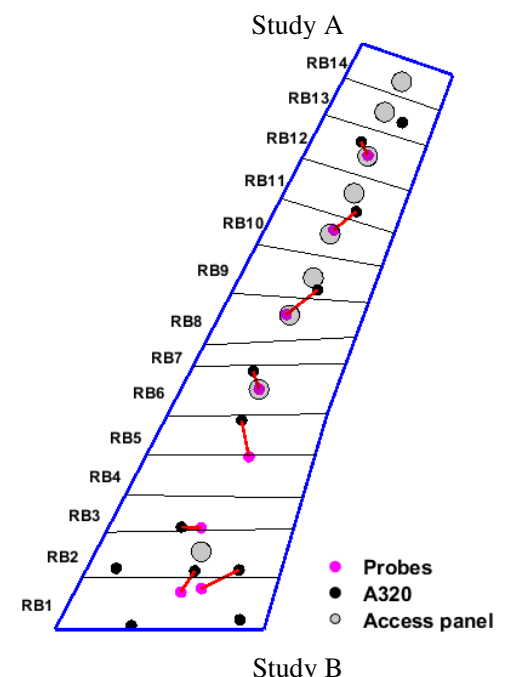

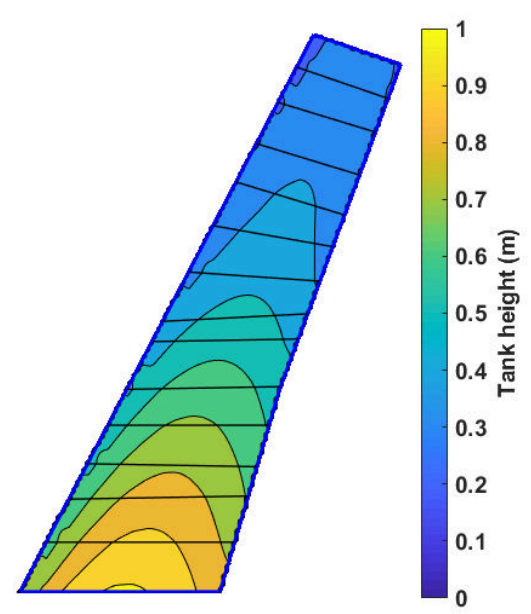

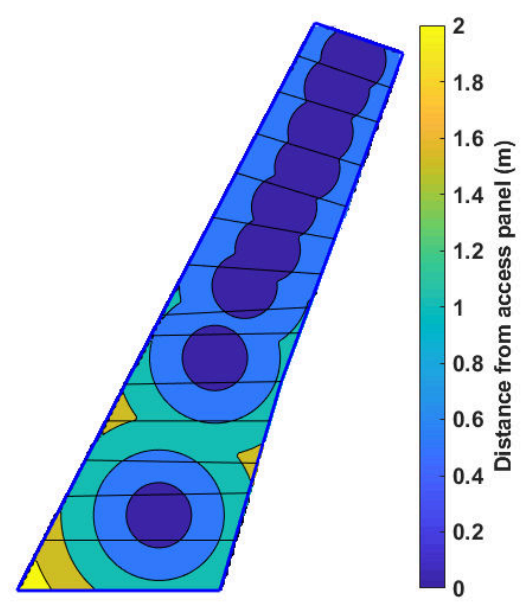

Fig. 15. FQI-GA and A320 probe position comparison and objective function grid values.

Fig. 15 also compares the A320 probe positions and highlights differences with the closest probes of the re-design. The A320 arrangement favours positions closer to access panels, rather than optimizing towards shorter probes.

A comparison of probe architecture measurement accuracy for studies A, B and the baseline, is shown in Fig. 16. It confirms that the FQI-GA solution has achieved constraint convergence, as the maximum error bounds for the three flight cases are within the respective requirement area. A difference between the resulting re-designed and the A320 architectures, is a spike in measurement error around the empty and full cases for the extended flight attitude case. 
The fuel-ullage interface surfaces for these cases, have no active probes, whereas the A320 achieves full coverage for the full tank study case set. RB1 always requires two probes, one for each redundant set, which is also the case for the A320 arrangement. The re-designed solutions, however, have probes towards the centre of the rib bay, whereas the A320 locates them close to the root rib. Their position indicates that they may be designed to cover cases below the assumed $0.1 \%$ unusable fuel limit of the available tank volume in the nominal case. Therefore, it is possible that the A320 design requirements were more stringent for the nominal tank study cases, as assumed for the re-design study.
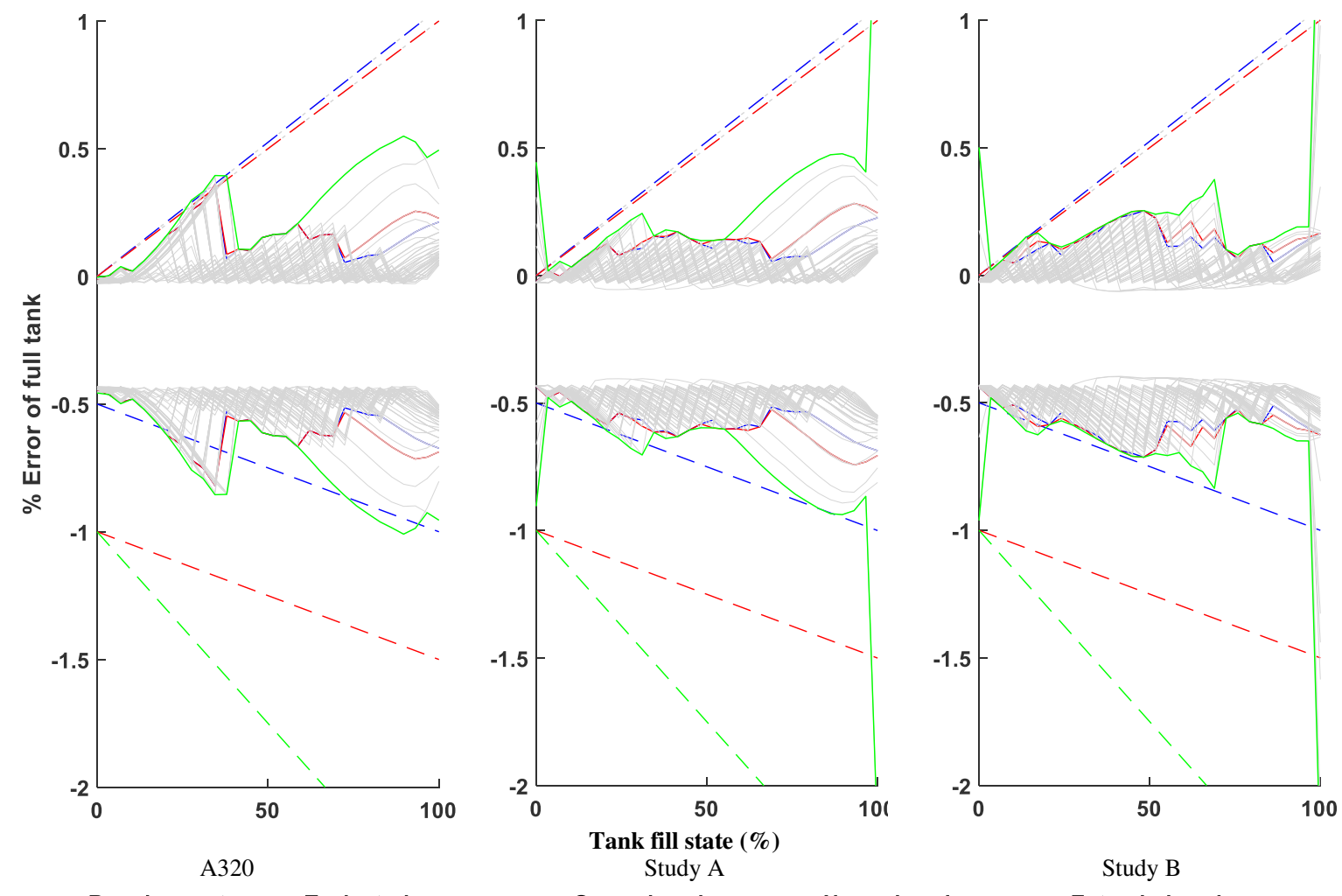

Requirement Tank study cases

Ground evelope

Normal evelope

Study B

Fig. 16. Comparison between A320 Study A and B probe architecture accuracies.

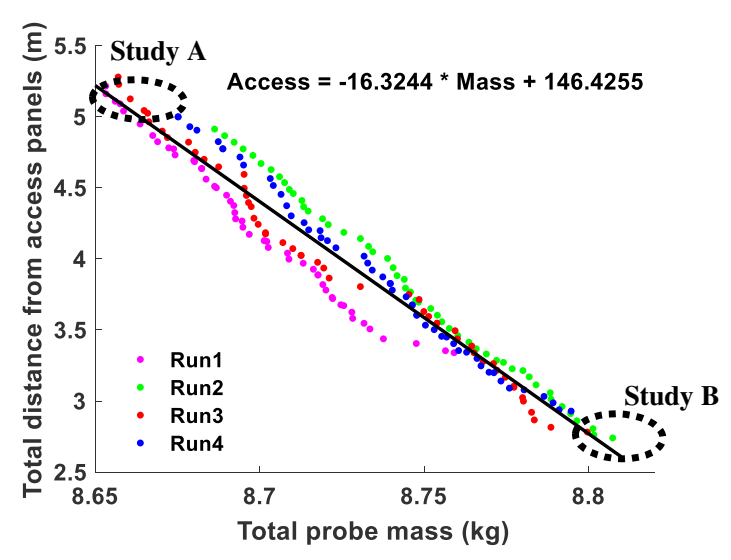

Fig. 17. Pareto fronts of four FQI-GA executions for study C. Probe mass is modelled as a function of probe length with 0.45 $\mathrm{kg} / \mathrm{m}$ and a base mass per probe of $0.8 \mathrm{~kg}$.

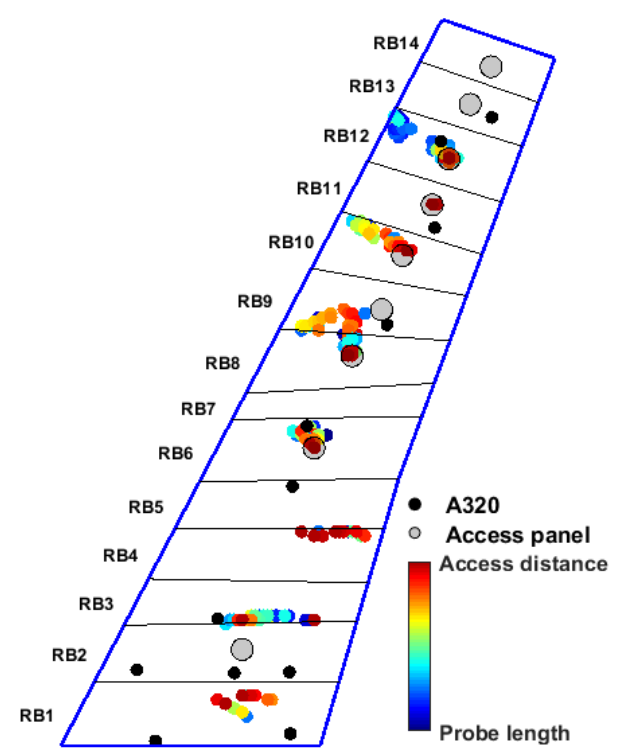

Fig. 18. Probe positions of all Pareto optimal FQI architectures for study $C$. 
Study $\mathrm{C}$ has two performance objectives and its resulting Pareto fronts are shown in Fig. 17. The four independent FQI-GA executions produced similar fronts with minor differences in accuracy and diversity. Overall an almost linear front, with a ratio of approximately -16 to 1 for access distance to probe length is estimated. This means that probe architectures will decrease 16 times faster for access distance than for the increase in probe mass. The shape of the Pareto front is mainly driven by the tank geometry height distribution, as shown in Fig. 15, whereas the gradient depends on the probe mass model.

Fig. 18 shows the probe positions of all Paretooptimal FQI architectures, shaded according to their objective values. At the extreme ends, the resulting probe positions mirror the position trends from study $\mathrm{A}$ and $\mathrm{B}$, as expected. Architectures which fall in between the two objective cases have probes which change position in various ways. Probe positions remain relatively static for RB1 and RB6 whereas other probes shift in the chord direction.

Even-though the exact probe locations of the A320 FQI design could not be regenerated by the FQI-GA process, results show good similarity in position trends. Factors impacting the FQI-GA outcomes have been discussed and range from accuracy requirement assumptions to tank geometry modelling. In order to select a probe architecture from the Pareto front, other requirements need to be considered. Effects such as installation interface availability on rib, spar or skin structures, proximity to water collection areas and other fuel system components need to be considered.

Rather than producing such a fully validated FQI design, the main outcome from the case study is the demonstration that the FQI-GA process can incorporate industrially representative requirements for multiple accuracy constraints, complex tank geometries and handle multiple design objectives.

\section{Conclusions}

This paper presents a new, knowledge-based engineering compatible solution process to the optimal placement of FQI gauging probes under redundancy and measurement accuracy constraints. It enables fast preliminary design studies, where objectives, constraints, requirements, and geometry can be explored.

The FQI NPP is formally defined, based on discretized tank geometry and fuel surfaces, projected onto a $2 \mathrm{D}$ plane. This converts the problem into a combinatorial set selection optimization problem, which is different to the traditional NPP. A genetic algorithm with a sequential, two-staged fitness assignment strategy is proposed and tested for a range of crossover procedures. It is shown that the treatment of probe sets according to their accuracy constraint convergence is key for any crossover procedure. A range of crossover methods for NPPs were tested, including subarea-swap, and are shown to only provide little performance benefits, compared with a baseline of local and global mutation operations only. The NSGA-II based fitness assignment operation provides a relatively evenly spaced final Pareto front for the two-objective problem. However as more objectives are considered, newer methods, such as MOE/D and NSGA-III need to be investigated.

The algorithm was tested with a real-world engineering case study of the re-design of an FQI probe architecture for estimated accuracy requirements and inboard wing tank geometry of the Airbus A320. The resulting Pareto front is linear, with an approximate slope of -16 to 1 between probe-to-access panel distance and total probe mass. It was also found that Pareto-optimal architectures have 8 probes, which is a reduction of 4 over the baseline. Resulting probe positions are comparable to the A320 baseline for an optimization of access only. Further work is required to model flap valves in the tank study and include varying discretization levels of both the geometry and the tank study cases, to establish their impact on the optimal probe positions.

\section{References}

1. SAE International. Aerospace Recommended Practice 4754A, "Guidelines for Development of Civil Aircraft and Systems", SAE; (2010)

2. Dean, S., R., H., Doherty, J., J., Wallace, T., R., "MDOBased Concept Modelling and the impact of fuel systems on wing design", 47 $7^{\text {th }}$ AIAA Aerospace Sciences Meeting, (2009)

3. Gavel., H., Oelvander. J., Krus, P., “Aircraft fuel system synthesis aided by interactive morphology, optimization and probabilistic design", 46th AIAA Aerospace Sciences Meeting and Exhibit, (2008)

4. Mihalyi, L., "Multi objective optimization and probabilistic design on aircraft fuel system", Master Thesis, Lulea Tekniska University, (2007)

5. Tookey, R M; Spicer, M G; Diston, D., "Integrated Design and Analysis of an Aircraft Fuel System", NATO/RTO Air Vehicle Technology Panel Symposium, Paris, France. Published in RTO-MP-089, (2003)

6. Dorbath, F., Nagel, B., Gollnick, V., "A Knowledge based approach for automated modelling of extended wing structures in preliminary aircraft design", $28^{\text {th }}$ ICAS, (2012)

7. La Rocca, G., "Knowledge based Engineering techniques to support aircraft design and optimization", $\mathrm{PhD}$ Thesis, TU Delft, (2011)

8. López, A., S., Munulury, .R., C., "Parametric modelling of aircraft fuel systems integration in rapid", $6^{\text {th }}$ EASN International Conference on Innovation in European Aeronautics Research, (2016)

9. Munulury, R., C., "Knowledge-based Aircraft Systems Integration", MODPROD Workshop, (2017)

10. Pearson, D.B., "A Capacitance-Type Fuel-Measurement System for Aircraft", Transactions of the American Institute of Electrical Engineers, Vol 66, Issue 1, (1947) 
11. SAE International. Aerospace Information Report AIR5691TM, "Guidance for the Design and Installation of Fuel Quantity Indicating Systems", SAE; (2013)

12. Langton, R. Clark, C., Hewitt, M., Richards, L., "Aircraft Fuel Systems", Wiley $1^{\text {st }}$ Edition, (2009)

13. Newport, R., Nelson, D., Manfred, M., "Digital fuel quantity indicating system for aircraft", AIAA - Digital avionics Systems Conference, (1984)

14. Fisher, H., "Re-profiled Fuel Quantity capacitance Probes for improved A300 FQI Accuracy", FAST Airbus technical digest magazine, (1984)

15. Zhang, Y.-H., Gong, Y.-J., Gu, T.-L., Li, Y. and Zhang, J., "Flexible genetic algorithm: A simple and generic approach to node placement problems", Applied Soft Computing, 52, pp. 457-470. (2017) (doi:10.1016/j.asoc.2016.10.022)

16. S. Abdollahzadeh, N.J. Navimipour, "Deployment strategies in the wireless sensor network: a comprehensive review", Comput. Commun.91-92, 1-16 (2016)

17. Mallardo, V., Aliabadi, M. "Optimal Sensor Placement for Structural, Damage and Impact Identification: A Review". Structural Durability \& Health Monitoring, 9(4), 287-323. (2013)

18. J. Seok, J. Lee, W. Kim and J. Lee, "A Bipopulation-Based Evolutionary Algorithm for Solving Full Area Coverage Problems", IEEE Sensors Journal, vol. 13, no. 12, pp. 47964807, (2013)

19. Rangel, E., \& Costa, D., \& Loula, A., "On redundant coverage maximization in wireless visual sensor networks: Evolutionary algorithms for multi-objective optimization", Applied Soft Computing. Vol. 82. 105578 (2019)

20. Khanali, M., Ahmadzadegan, S., Omid, M. et al., "Optimizing layout of wind farm turbines using genetic algorithms in Tehran province, Iran.", Int J Energy Environ Eng 9, 399-411 (2018) https://doi.org/10.1007/s40095018-0280-X

21. Charhouni, N., Sallaou, M. \& Mansouri, K.,"Realistic wind farm design layout optimization with different wind turbines types", Int J Energy Environ Eng 10, 307-318 (2019). https://doi.org/10.1007/s40095-019-0303-2

22. Y. Chen, H. Li, K. Jin, Q. Song, "Wind farm layout optimization using genetic algorithm with different hub height wind turbines". Energy Convers. Manag. 70, 56-65 (2013)

23. Thiene, M., Sharif Khodaei, Z., Aliabadi, M. H., "Optimal sensor placement for maximum area coverage (MAC) for damage localization in composite structures" Smart Materials and Structures, Vol. 25 No.9 (2016)

24. Ismail, Z., Mustapha, S., Fakih, M. A., \& Tarhini, H., 'Sensor placement optimization on complex and large metallic and composite structures. ", Structural Health Monitoring, 19(1), 262-280.

(2020). https://doi.org/10.1177/1475921719841307

25. Eiben, A. E., \& Smith, J. E., "Introduction to evolutionary computing", $2^{\text {nd }}$ Edition, Springer-Verlag (2015)

26. Coello, C., Lamont, G., Van Veldhuizen, D., "Evolutionary Algorithms for Solving Multi-Objective Problems", Springer Verlag, $2^{\text {nd }}$ Edition, (2007)

27. Wang, Q., Liu, H., Yuan, J., \& Chen, L. “Optimizing the energy-spectrum efficiency of cellular systems by evolutionary multi-objective algorithm." Integrated Computer-Aided Engineering, 26(2), 207-220. (2019)

28. Wei, Y, Jin, JG, Yang, J, Lu, L. Strategic network expansion of urban rapid transit systems: A bi-objective programming model. Computer-Aided Civil and Infrastructure Engineering.; Vol. 34: pp431- 443. (2019) https://doi.org/10.1111/mice.12426

29. Mencía, C., Sierra, M.R., Mencía, R., and Varela, R., "Evolutionary one-machine scheduling in the context of electric vehicles charging", Integrated Computer-Aided Engineering, 26:1, pp. 49-63. (2019)

30. Palacios, J.J., González-Rodríguez, I., Vela, C.R., Puente, J., "Satisfying flexible due dates in fuzzy job shop by means of hybrid evolutionary algorithms", Integrated ComputerAided Engineering, 26:1, pp. 65-84. (2019)
31. Frank, C., P., Marlier, R., A., Pinon-Fisher, O., J., Mavris, D., $\mathrm{N}$., "Evolutionary multi-objective multi architecture design space exploration methodology", Optimization Engineering, Vol 19, pp 359-381, (2018)

32. Judt, D., M., Lawson, C., P., "Application of an automated aircraft architecture generation and analysis tool to unmanned aerial vehicle subsystem design", Proceedings of the Institution of Mechanical Engineers, Part G: Journal of Aerospace Engineering, 229(9), 1690-1708, (2015)

33. Judt, D. M. and Lawson, C., "Development of an automated aircraft subsystem architecture generation and analysis tool", Engineering Computations, Vol. 33, No. 5, pp. 13271352, (2016)

34. Ho-Huu, V.; Hartjes, S.; Visser, H.G.; Curran, R. “An Efficient Application of the MOEA/D Algorithm for Designing Noise Abatement Departure Trajectories". Aerospace, Vol 4, 54. (2017)

35. Zhang, M., Filippone, A., Bojdo, N., "Multi-objective optimization of aircraft departure trajectories", Aerospace science and technology, Vol. 79, 37-47, (2018)

36. K. Deb, A. Pratap, S. Agarwal, and T. Meyarivan, "A fast and elitist multi-objective genetic algorithm: NSGA-II", IEEE Transactions on Evolutionary Computation, vol. 6, no. 2, pp. 182-197, (2002)

37. Zitzler, E., Thiele, L., “An Evolutionary Algorithm for Multiobjective Optimization: The Strength Pareto Approach". Technical Report 43, Computer Engineering and Communication Networks Lab (TIK), Swiss Federal Institute of Technology (ETH), Zurich, Switzerland, (1998)

38. Zhang, Q., and Li, H., "MOEA/D: A Multiobjective Evolutionary Algorithm Based on Decomposition," in IEEE Transactions on Evolutionary Computation, vol. 11, no. 6, pp. 712-731, Dec. (2007), doi: 10.1109/TEVC.2007.892759.

39. Deb, K. and Jain H., "An Evolutionary Many-Objective Optimization Algorithm Using Reference-Point-Based Nondominated Sorting Approach, Part I: Solving Problems With Box Constraints," in IEEE Transactions on Evolutionary Computation, vol. 18, no. 4, pp. 577-601, Aug. (2014), doi: 10.1109/TEVC.2013.2281535.

40. S. Kukkonen and J. Lampinen, "GDE3: the third evolution step of generalized differential evolution," 2005 IEEE Congress on Evolutionary Computation, Edinburgh, Scotland, 2005, pp. 443-450 Vol.1, doi: 10.1109/CEC.2005.1554717.

41. B. Saavedra-Moreno, S. Salcedo-Sanz, A. Paniagua-Tineo, L. Prieto, A. Portilla-Figueras, "Seeding evolutionary algorithms with heuristics for optimal wind turbines positioning in wind farms", Renew. Energy, Vol. 36, pp2838-2844. (2011)

42. J.S. González, A.G.G. Rodriguez, J.C. Mora, J.R. Santos, M.B. Payan, "Optimization of wind farm turbines layout using an evolutive algorithm", Renew. Energy Vol. 35, pp1671-1681, (2010)

43. Ryerkerk, M., Averill, R., Deb, K. et al. "A survey of evolutionary algorithms using metameric representations", Genet Program Evolvable Mach 20, 441-478 (2019). https://doi.org/10.1007/s10710-019-09356-2

44. Wagner M., Neumann F., "Parsimony Pressure versus Multi-objective Optimization for Variable Length Representations", In: Coello C.A.C., Cutello V., Deb K., Forrest S., Nicosia G., Pavone M. (eds) Parallel Problem Solving from Nature - PPSN XII. PPSN (2012). Lecture Notes in Computer Science, vol 7491. Springer, Berlin, Heidelberg

45. Autoflug, "Fuel Gauge transmitter datasheet", www.autoflug.de, accessed 2020

46. Kramer, O. "A Review of Constraint-Handling Techniques for Evolution Strategies”, Applied Comp. Int. Soft Computing. (2010) 10.1155/2010/185063.

47. E. Zitzler and L. Thiele "Multiobjective evolutionary algorithms: a comparative case study and the strength pareto approach" evolutionary computation IEEE transactions on vol. 3 no. 4 pp. 257-271 (1999)

48. N. Riquelme, C. Von Lücken and B. Baran, "Performance metrics in multi-objective optimization," 2015 Latin 
American Computing Conference (CLEI), Arequipa, 2015, pp. 1-11, doi: 10.1109/CLEI.2015.7360024.

49. Rostami, S., Neri, F. \& Gyaurski, K., "On Algorithmic Descriptions and Software Implementations for Multiobjective Optimisation: A Comparative Study." SN COMPUT. SCI. 1, 247 (2020).

50. Rostami, S. and Neri, F. "Covariance Matrix Adaptation Pareto Archived Evolution Strategy with Hyper volumesorted Adaptive Grid Algorithm". Integrated ComputerAided Engineering, 23 (4), pp. 313-329, (2016)

51. Rostami, S. and Ferrante Neri. "A fast hypervolume driven selection mechanism for many-objective optimisation problems." Swarm Evol. Comput. Vol.34, pp50-67. (2017)

52. Obert, E., "Aerodynamic design of transport aircraft", IOS Press, (2009)

53. Orlita, M., \& Vos, R. "Cruise Performance Optimization of the Airbus A320 through Flap Morphing”. In 17th AIAA Aviation Technology, Integration, and Operations Conference, (2017),DOI: 10.2514/6.2017-3264

54. Gresty, D., Mueller, J., Rudolph, P., Schallert, C., "Airplane wing with slat and Krueger flap -EP1338506A1”, European Patent Office, (2002)

55. Hahn, A., "Vehicle Sketch Pad: Parametric Geometry for Conceptual Aircraft Design", 48th AIAA Aerospace Sciences Meeting, Orlando, FL, Jan 4 - 7, AIAA-2010-657, (2010)

56. Walker, R., “The new A340-500/600 Fuel System”, FAST Airbus technical digest magazine, (2000)

57. Vallée, G., Pernet, R., Urdiroz, A., "Fuel monitoring on A320 Family aircraft “. The Airbus Safety Magazine, issue 22, (2015)

58. Airbus. "Operational Liaison Meeting FBW Aircraft: Fuel Accuracy". Airbus; (2017)

59. European Aviation Safety Agency, "Certification Specifications and acceptable means of compliance for large aeroplanes - CS-25 amendment 22", \$25.1337, (2018)

60. Airbus, A318/A319/A320/A321, "Flight crew operating manual", Airbus; (2017)

61. Airbus, "Getting to grips with aircraft performance", Airbus flight operations support \& line assistance magazine, (2002)

62. Hilmi, M., H., R., "Investigating pressure-based aircraft fuel gauging systems", MSc Thesis, Cranfield University, (2018)

63. SAE International. Aerospace Information Report AIR1 184TM, "Capacitive Fuel Gauging System Accuracies", SAE; (2016) 
Rapid design of aircraft fuel quantity indication systems via multi-objective evolutionary algorithms

Judt, David M.

IOS Press

Judt D, Lawson C, van Heerden ASJ. (2020) Rapid design of aircraft fuel quantity indication systems via multi-objective evolutionary algorithms. Integrated Computer-Aided Engineering, Volume 28, Issue 2, 2021, pp. 141-158

https://doi.org/10.3233/ICA-200646

Downloaded from Cranfield Library Services E-Repository 